Article

\title{
Fixed Point Results on $\Delta$-Symmetric Quasi-Metric Space via Simulation Function with an Application to Ulam Stability
}

\author{
Badr Alqahtani ${ }^{1}$, Andreea Fulga ${ }^{2}$ and Erdal Karapınar ${ }^{3,4, * \mathbb{D}}$ \\ 1 Department of Mathematics, King Saud University, P.O. Box 2455, Riyadh 11451, Saudi Arabia; \\ balqahtani1@ksu.edu.sa \\ 2 Department of Mathematics and Computer Sciences, Universitatea Transilvania Brasov, 500036 Brasov, \\ Romania; afulga@unitbv.ro \\ 3 Department of Mathematics, Atilim University, 06836 Ankara, Turkey \\ 4 Department of Medical Research, China Medical University, Taichung 40402, Taiwan \\ * Correspondence: erdalkarapinar@yahoo.com
}

Received: 8 September 2018; Accepted: 13 October 2018; Published: 17 October 2018

\begin{abstract}
In this paper, in the setting of $\Delta$-symmetric quasi-metric spaces, the existence and uniqueness of a fixed point of certain operators are scrutinized carefully by using simulation functions. The most interesting side of such operators is that they do not form a contraction. As an application, in the same framework, the Ulam stability of such operators is investigated. We also propose some examples to illustrate our results.
\end{abstract}

Keywords: quasi-metric space; $\Delta$-symmetry; fixed point theorems; metric space

MSC: 47H10; 54H10

\section{Introduction}

In the last few decades, one of the hot topics in topology and analysis has been the quasi-metric, which is a natural generalization of the notion of the metric; see, e.g., [1-4]. Roughly speaking, the quasi-metric appears to be obtained by relaxing the symmetric condition from the axioms of the standard metric. Regarding the physical phenomena, the quasi-metric can be more useful to consider in solving real-life problems [5]. On the other hand, one can ask an impulsive question about whether there is a positive constant $\Delta$ such that the distance from a point $p$ to $q$ is dominated by $\Delta$ times the distance from $q$ to $p$. The answer is affirmative, and such spaces are called the $\Delta$-symmetric quasi-metric. These spaces are quite rich and lie between the quasi-metric and metric.

Quasi-metric spaces are very interesting topics for researchers who work in fixed point theory; see, e.g., [6,7] and the references therein. It is an indispensable fact that real-life applications of fixed point theory have a wide range. Indeed, the border of the range is beyond the following question: Do we transfer the real-life problems in the form of $f(x)=x$ or not? Consequently, after the first metric fixed point result of Banach, several authors have reported a number of interesting results in various directions. Here, we mention one of the interesting generalization of the Banach contraction mapping principle that was given by Seghal [8].

Theorem 1. ([8]) Let $(\mathcal{M}, d)$ be a complete metric space and $T$ a continuous self-mapping of $\mathcal{M}$, which satisfies the condition that there exists a real number $q, 0<q<1$ such that for each $v \in \mathcal{M}$, there exists a positive integer $m(v)$ such that for each $w \in \mathcal{M}$,

$$
d\left(T^{m(v)} v, T^{m(v)} w\right) \leq q d(v, w) .
$$


Then, $T$ has a unique fixed point in $\mathcal{M}$.

This result above was improved by Guseman [9] by removing the continuity condition. Later, K.Iseki [10], J. Matkowski [11], Singh [12] and Ray and Rhoades [13] extended the result of Seghal [8]. The result of Kincses and Totik [14] is one of the most improved results in this direction.

Theorem 2. ([14]) Let $T$ be a self-mapping on a metric space $(\mathcal{M}, d)$ such that for some $q \in[0,1)$ and for all $v, w \in \mathcal{M}$, we can find a positive integer $m(v)$ such that:

$$
d\left(T^{m(v)} v, T^{m(v)} w\right) \leq q \max \left\{d(v, w), d\left(w, T^{m(v)} v\right), d\left(v, T^{m(v)} w\right)\right\}
$$

Then, T has a unique fixed point $v^{*}$.

Theorem 3. ([14]) Let $T$ be a self-map of a metric space $(\mathcal{M}, d)$. Assume there exists a nonincreasing function $\beta:(0, \infty) \rightarrow[0,1)$ such that for each $v \in \mathcal{M}$, there exists a positive integer $m(v)$ such that for each $w \in \mathcal{M}$ with $w \neq v$,

$$
d\left(T^{m(v)} v, T^{m(v)} w\right) \leq \beta[d(v, w)] d(v, w) .
$$

Then, $T$ has a unique fixed point $v^{*}$.

The inflation of so many results causes a commotion. Therefore, it is natural to consider combining and unifying the existence results. The notion of the simulation function is one of the successful consequences of this approach. By using the simulation function, it is possible to combine several distinct types of contractions and hence unify a number of existing results in a single theorem.

In this paper, we aim to get not only the most general metric fixed point results in the context of quasi-metric space, but also unify the several existing results in this direction, including the results of Seghal [8], Guseman [9], Kincses and Totik [14].

\section{Preliminaries}

We denote the set of non-negative reals by $\mathbb{R}_{0}^{+}$.

Definition 1. For $\mathcal{M} \neq \varnothing$, a function $q: \mathcal{M} \times \mathcal{M} \rightarrow \mathbb{R}_{0}^{+}$is called quasi-metric if the following assumptions are held:

$$
\begin{aligned}
& q(s, t)=q(t, s)=0 \Leftrightarrow s=u ; \\
& q(s, u) \leq q(s, t)+q(t, u), \text { for all } s, t, u \in \mathcal{M} .
\end{aligned}
$$

Here, the pair $(\mathcal{M}, q)$ is called a quasi-metric space.

The quasi-metric notion is a concrete extension of the metric concept. Therefore, as expected, each metric space forms a quasi-metric space, but the converse is not necessarily true. For instance, the well-known functions $l, r: \mathbb{R} \times \mathbb{R} \rightarrow \mathbb{R}_{0}^{+}$, defined by $l(s, t)=\max \{s-t, 0\}$ and $r(s, t)=\max \{t-s, 0\}$, are quasi-metric, but not a metric. Indeed, $d(s, t):=\max \{l(s, t), r(s, t)\}$ forms a standard Euclidean metric on $\mathbb{R}$.

A sequence $\left\{s_{n}\right\}$ in $\mathcal{M}$ converges to $s \in \mathcal{M}$ if:

$$
\lim _{n \rightarrow \infty} q\left(s_{n}, s\right)=\lim _{n \rightarrow \infty} q\left(s, s_{n}\right)=0 .
$$

In a quasi-metric space $(\mathcal{M}, q)$, the limit for a convergent sequence is unique. If $s_{n} \rightarrow s$, we have for all $s \in \mathcal{M}$ :

$$
\lim _{n \rightarrow \infty} q\left(s_{n}, t\right)=q(s, t) \text { and } \lim _{n \rightarrow \infty} q\left(t, s_{n}\right)=q(t, s)
$$


Let $(\mathcal{M}, q)$ be a quasi-metric space and $\left\{s_{n}\right\}$ be a sequence in $\mathcal{M}$. We say that a sequence $\left\{s_{n}\right\}$ is left-Cauchy if for every $\epsilon>0$, there exists a positive integer $N=N(\epsilon)$ such that $q\left(s_{n}, s_{m}\right)<\epsilon$ for all $n \geq m>N$. Analogously, a sequence $\left\{s_{n}\right\}$ is called right-Cauchy if for every $\epsilon>0$, there exists a positive integer $N=N(\epsilon)$ such that $q\left(s_{n}, s_{m}\right)<\epsilon$ for all $m \geq n>N$. Furthermore, the sequence $\left\{s_{n}\right\}$ is said to be Cauchy if for every $\epsilon>0$, there exists a positive integer $N=N(\epsilon)$ such that $q\left(s_{n}, s_{m}\right)<\epsilon$ for all $m, n>N$. It is evident that a sequence $\left\{s_{n}\right\}$ in a quasi-metric space is Cauchy if and only if it is left-Cauchy and right-Cauchy. A quasi-metric space $(\mathcal{M}, q)$ is left-complete (respectively, right-complete, complete) if each left-Cauchy sequence (respectively, right-Cauchy sequence, Cauchy sequence) in $\mathcal{M}$ is convergent.

Definition 2. Suppose that $(\mathcal{M}, q)$ is a quasi-metric space, and $\left\{s_{n}\right\} \subset \mathcal{M}$ and $s \in \mathcal{M}$. Let $T$ be a self-mapping. We say that $T$ is:

(i) right-continuous if $q\left(T s_{n}, T s\right) \rightarrow 0$ whenever $q\left(s_{n}, s\right) \rightarrow 0$;

(ii) left-continuous if $q\left(T s, T s_{n}\right) \rightarrow 0$ whenever $q\left(s, s_{n}\right) \rightarrow 0$;

(iii) continuous if $\left\{T_{n}\right\} \rightarrow$ Ts whenever $\left\{s_{n}\right\} \rightarrow s$.

Observe that the simultaneous right and left continuity of a mapping yields the continuity of it.

Definition 3. Suppose that $(\mathcal{M}, q)$ is a quasi-metric space. We say that it is $\Delta$-symmetric if there exists a positive real number $\Delta>0$ such that:

$$
q(t, s) \leq \Delta \cdot q(s, t) \text { for all } s, t \in \mathcal{M}
$$

It is clear that if $\Delta=1$, then the $\Delta$-symmetric quasi-metric space $(\mathcal{M}, q)$ forms a metric space.

Example 1. Suppose that $(\mathcal{M}, q)$ is a quasi-metric space, and a function $q: \mathcal{M} \times \mathcal{M} \rightarrow \mathbb{R}_{0}^{+}$is defined as follows:

$$
q(s, t)=\left\{\begin{aligned}
3 \cdot d(s, t) & \text { if } s \geq t \\
d(s, t) & \text { otherwise }
\end{aligned}\right.
$$

$(\mathcal{M}, q)$ is a three-symmetric quasi-metric space, but it is not a metric space.

In the following, we recall the main properties of $\Delta$-symmetric quasi-metric spaces.

Lemma 1. (See, e.g., [15]) Let $(\mathcal{M}, q)$ be a $\Delta$-symmetric quasi-metric space and $\left\{s_{n}\right\}$ be a sequence in $\mathcal{M}$ and $s \in \mathcal{M}$. Then:

(i) $\left\{s_{n}\right\}$ right-converges to $s \Leftrightarrow\left\{s_{n}\right\}$ left-converges to $s \Leftrightarrow\left\{s_{n}\right\}$ converges to $x$.

(ii) $\quad\left\{s_{n}\right\}$ is right-Cauchy $\Leftrightarrow\left\{s_{n}\right\}$ is left-Cauchy $\Leftrightarrow\left\{s_{n}\right\}$ is Cauchy.

(iii) If $\left\{t_{n}\right\}$ is a sequence in $\mathcal{M}$ and $q\left(s_{n}, t_{n}\right) \rightarrow 0$, then $q\left(t_{n}, s_{n}\right) \rightarrow 0$.

A non-decreasing function $\psi: \mathbb{R}_{0}^{+} \rightarrow \mathbb{R}_{0}^{+}$is called a comparison function (see, e.g., [16]) if:

$(\Omega) \lim _{n \rightarrow \infty} \psi^{n}(t)=0$, for $t \in \mathbb{R}_{0}^{+}$.

Proposition 1. (See, e.g., [16]) Let $\psi: \mathbb{R}_{0}^{+} \rightarrow \mathbb{R}_{0}^{+}$be a comparison function. Then, we have:

(A) $\quad \psi$ is continuous at zero.

(B) For $k \in \mathbb{N}$, each $\psi^{k}$ also forms a comparison function.

(Г) $\quad \psi(t)<t$ for all $t>0$.

A non-decreasing function $\psi: \mathbb{R}_{0}^{+} \rightarrow \mathbb{R}_{0}^{+}$is called a c-comparison if 


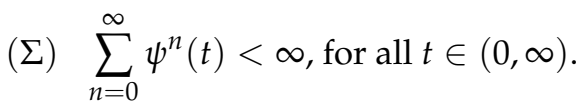

We reserve the letter $\Psi$ to denote the family of all c-comparison functions. Note that each $c$-comparison function forms a comparison function. For more details and examples for comparison and $c$-comparison functions, see, e.g., $[16,17]$.

Let $\varphi: \mathbb{R}_{0}^{+} \rightarrow \mathbb{R}_{0}^{+}$be a $c$-comparison function that satisfies the following condition:

(घ) $\lim _{n \rightarrow \infty}(t-\varphi(t))=\infty$.

We shall use the letter $\Phi$ to represent the class of such functions (i.e., the set of all c-comparison functions that satisfies the condition $(\Xi)$ ).

In what follows, we recollect the definition of the simulation function.

Definition 4. See, e.g., [18]. Suppose that $\zeta: \mathbb{R}_{0}^{+} \times[0, \infty) \rightarrow \mathbb{R}$ fulfills:

$\left(\zeta_{1}\right) \zeta(t, s)<s-t$ for all $t, s>0$;

$\left(\zeta_{2}\right)$ if $\left\{t_{n}\right\},\left\{s_{n}\right\}$ are sequences in $(0, \infty)$ such that $\lim _{n \rightarrow \infty} t_{n}=\lim _{n \rightarrow \infty} s_{n}>0$, then:

$$
\limsup _{n \rightarrow \infty} \zeta\left(t_{n}, s_{n}\right)<0
$$

Then, $\zeta$ is called a simulation function. Further, the letter $\mathcal{S}$ denotes all simulation functions $\zeta$.

We underline the fact that the condition $\zeta(0,0)=0$ is superfluous. Due to the axiom $\left(\zeta_{1}\right)$, we have:

$$
\zeta(t, t)<0 \text { for all } t>0 .
$$

For more details and examples on simulation functions, see, e.g., [6,7,19-22].

The notions of an $\alpha$-admissible mapping [23] and triangular $\alpha$-admissible mappings [24] were refined by Popescu [25] as follows:

Definition 5. [25] Let $\alpha: X \times X \rightarrow \mathbb{R}_{0}^{+}$be a mapping and $\mathcal{M} \neq \varnothing$. A self-mapping $T: \mathcal{M} \rightarrow \mathcal{M}$ is said to be an $\alpha$-orbital admissible if for all $s \in \mathcal{M}$, we have:

$$
\alpha(s, T s) \geq 1 \Rightarrow \alpha\left(T s, T^{2} s\right) \geq 1 .
$$

Furthermore, $\alpha$-orbital admissible mapping $T$ is called triangular $\alpha$-orbital admissible if the following condition holds:

(TO) $\alpha(s, t) \geq 1$ and $\alpha(s, T t) \geq 1$ implies that $\alpha(s, T t) \geq 1$, for all $s, t \in \mathcal{M}$.

Each $\alpha$-admissible mapping is an $\alpha$-orbital admissible mapping. For more details and interesting examples, see, e.g., [21,26-35].

A set $\mathcal{M}$ is regular with respect to mapping $\alpha: \mathcal{M} \times \mathcal{M} \rightarrow \mathbb{R}_{0}^{+}$if $\left\{s_{n}\right\}$ is a sequence in $\mathcal{M}$ such that $\alpha\left(s_{n}, s_{n+1}\right) \geq 1$ for all $n$ and $s_{n} \rightarrow s \in \mathcal{M}$ as $n \rightarrow \infty$, then $\alpha\left(s, s_{n}\right) \geq 1$ for all $n$.

The following technical lemma will be used in the proof of the first main result of this paper.

Lemma 2. Let $\mathcal{M}$ be a non-empty set and $T: \mathcal{M} \rightarrow \mathcal{M}$ form a triangular $\alpha$-orbital admissible mapping. Consider the iterative sequence $s_{n}=T s_{n-1}, n \in \mathbb{N}$. If there exists $s_{0} \in \mathcal{M}$ such that $\alpha\left(s_{0}, T s_{0}\right) \geq 1$, then for any $k \in \mathbb{N}$, we have:

$$
\alpha\left(T^{k-1} s_{0}, T^{k} s_{0}\right) \geq 1
$$

and:

$$
\alpha\left(s_{k}, T^{p} s_{k}\right) \geq 1,
$$

for all $p \in\{0,1,2, \ldots\}$. 
Proof. Due to the statement of the theorem, there exists a point $s_{0} \in \mathcal{M}$ such that $\alpha\left(s_{0}, T s_{0}\right) \geq 1$. Based on the definition of the iterative sequence $\left\{s_{n}\right\} \subset \mathcal{M}$ and taking into account that $T$ is $\alpha$-orbital admissible, we get:

$$
\alpha\left(s_{0}, s_{1}\right)=\alpha\left(s_{0}, T s_{0}\right) \geq 1 \Rightarrow \alpha\left(T s_{0}, T^{2} s_{0}\right)=\alpha\left(s_{1}, s_{2}\right) \geq 1
$$

Recursively, we derive that:

$$
\alpha\left(s_{n-1}, s_{n}\right)=\alpha\left(T^{n-1} s_{0}, T^{n} s_{0}\right) \geq 1, \text { for all } n \in \mathbb{N}_{0}
$$

On the other side, using the condition (TO) of Definition 5, we deduce that:

$$
\begin{gathered}
\alpha\left(s_{k}, T s_{k}\right)=\alpha\left(s_{k}, s_{k+1}\right) \geq 1 \text { and } \alpha\left(s_{k+1}, s_{k+2}\right) \alpha\left(s_{k+1}, T s_{k+1}\right) \geq 1 \\
\text { implies that } \alpha\left(s_{k}, s_{k+2}\right)=\alpha\left(s_{k}, T^{2} s_{k}\right) \geq 1
\end{gathered}
$$

Recursively, for all $p \in\{1,2, \ldots\}$, we have:

$$
\alpha\left(s_{k}, T^{p} s_{k}\right) \geq 1 \text {, for any } k \in \mathbb{N} .
$$

Definition 6. Let $\alpha: \mathcal{M} \times \mathcal{M} \rightarrow \mathbb{R}_{0}^{+}$be a function. We say that a self-mapping $T: \mathcal{M} \rightarrow \mathcal{M}$ satisfies the condition (U) if:

$$
\alpha(u, v) \geq 1 \text { and } \alpha(v, u) \geq 1
$$

for all $u, v \in \operatorname{Fix}\left(T^{n}\right)=\left\{x \in \mathcal{M}: T^{n(u)} x=x\right\}$.

\section{Main Results}

We state our first main results.

Theorem 4. Let $(\mathcal{M}, q)$ be a complete $\Delta$-symmetric quasi-metric space, $T$ be a continuous self-mapping and $\alpha: \mathcal{M} \times \mathcal{M} \rightarrow \mathbb{R}_{0}^{+}, \zeta \in \mathcal{S}, \varphi \in \Psi$. Suppose that for every $x \in \mathcal{M}$, there is a positive integer $n=n(s)$ such that the inequality:

$$
\zeta\left(\alpha(s, t) q\left(T^{n(s)} s, T^{n(s)} t\right), \varphi(q(s, t))\right) \geq 0,
$$

is fulfilled for all $t \in \mathcal{M}$. Moreover, assume that:

(i) T forms a triangular $\alpha$-orbital admissible;

(ii) there exists $s_{0} \in \mathcal{M}$ with the property $\alpha\left(s_{0}, T s_{0}\right) \geq 1$ and $\alpha\left(T s_{0}, s_{0}\right) \geq 1$;

Then, $T$ has a fixed point $u \in \mathcal{M}$.

Proof. Fix $s \in \mathcal{M}$. By assumption, there exist $n=n(s)$ such that for all $t \in \mathcal{M}$ :

$$
\zeta\left(\alpha(s, t) q\left(T^{n(s)} s, T^{n(s)} t\right), \varphi(q(s, t))\right) \geq 0 .
$$

Regarding the condition $(\zeta 1)$, we find:

$$
\begin{aligned}
0 & \leq \zeta\left(\alpha(s, t) q\left(T^{n(s)} s, T^{n(s)} t\right), \varphi(q(s, t))\right) \\
& <\varphi(q(s, t))-\alpha(s, t) q\left(T^{n(s)} s, T^{n(s)} t\right)
\end{aligned}
$$

which yields that:

$$
\alpha(s, t) q\left(T^{n(s)} s, T^{n(s)} t\right) \leq \varphi(q(s, t)) .
$$


Now, we shall define $r_{1}(s)$ and $r_{2}(s)$ as follows:

$$
\begin{aligned}
& r_{1}(s)=\sup \left\{q\left(s, T^{m} s\right): m \in \mathbb{N}\right\} \\
& r_{2}(s)=\sup \left\{q\left(T^{m} s, s\right): m \in \mathbb{N}\right\}
\end{aligned}
$$

for any $s \in \mathcal{M}$.

In what follows, we shall show that $r_{1}(s)<\infty$ and $r_{2}(s)<\infty$.

First of all, for a given $p \in\{0,1, \ldots, n(s)-1\}$, we set $l=\max \left\{a_{0}, q\left(s, T^{n(s)} s\right)\right\}$ and:

$$
a_{m}=q\left(s, T^{\left.m n(s)+p_{s}\right)} \text { for all } m \in \mathbb{N}\right.
$$

Due to $(\Sigma)$, there exists $c \in(0, \infty)$, with $c>l$ such that $t-\varphi(t)>l$ for all $t \in[c, \infty)$. Notice that $c>l \geq a_{0}$.

Now, we claim that there exist $c \in(0, \infty)$, with $c>l$ such that:

$$
a_{m}<c \text { for all } m \in \mathbb{N} \text {. }
$$

Observe that the assumption (18) holds for $m=0$. On the contrary, we suppose that there is a positive integer $k$ so that:

$$
a_{k-1}<c \leq a_{k}
$$

Regarding the triangle inequality, together with the inequalities (16) and (12), we have that:

$$
\begin{aligned}
a_{k} & =q\left(s, T^{k \cdot n(s)+p_{s}} \leq q\left(s, T^{n(s)} s\right)+q\left(T^{n(s)} s, T^{k \cdot n(s)+p_{s}}\right)\right. \\
& =q\left(s, T^{n(s)} s\right)+q\left(T^{n(s)} s, T^{n(s)}\left(T^{\left.(k-1) \cdot n(s)+p_{s}\right)}\right)\right. \\
& \leq l+\alpha\left(s, T^{\left.(k-1) \cdot n(s)+p_{s}\right)} q\left(T^{n(s)} s, T^{n(s)}\left(T^{\left.(k-1) \cdot n(s)+p_{s}\right)}\right)\right.\right. \\
& \leq l+\varphi\left(q\left(s, T^{\left.(k-1) \cdot n(s)+p_{s}\right)}\right)\right. \\
& =l+\varphi\left(a_{k-1}\right) .
\end{aligned}
$$

Taking, the assumption (19) into account and keeping $\varphi \in \phi$ in mind, we get:

$$
a_{k}-\varphi\left(a_{k}\right) \leq l
$$

This is a contradiction, since we already supposed that $a_{k} \geq c$, and we have $a_{k}-$ $\varphi\left(a_{k}\right)>l$ due to $(\mathbb{E})$. Hence, the set $\left\{q\left(s, T^{m \cdot n(s)+p}\right): m \in \mathbb{N}\right\}$ is bounded. Due to fact that $p \in\{0,1, \ldots, n(s)-1\}$, we conclude that the set $\left\{q\left(s, T^{m} s\right): m \in \mathbb{N}\right\}$ is bounded, and so, $r_{1}(s)=\sup \left\{q\left(s, T^{m} s\right): m \in \mathbb{N}\right\}<+\infty$.

On the other hand, the space $(\mathcal{M}, q)$ is the $\Delta$-symmetric, so, for all $s \in \mathcal{M}$ and $m \in \mathbb{N}$, we have:

$$
q\left(T^{m} s, s\right) \leq C \cdot q\left(s, T^{m} s\right) \leq C \cdot r_{1}(s)
$$

From here, we conclude that the set $\left\{q\left(T^{m} s, s\right): m \in \mathbb{N}\right\}$ is also bounded and $r_{2}(s)<+\infty$. Therefore, for all $s \in \mathcal{M}$ and all $m_{1}, m_{2} \in \mathbb{N}$,

$$
q\left(T^{m_{1}} s, T^{m_{2}} s\right) \leq q\left(T^{m_{1}} s, s\right)+q\left(s, T^{m_{2}} s\right) \leq r_{1}(s)+r_{2}(s)<+\infty
$$

which shows that the orbit $\left\{T^{k_{S}}: k \in \mathbb{N}\right\}$ is a bounded subset of $\mathcal{M}$.

Now, let $s_{0} \in \mathcal{M}$ be an arbitrary point. If $T s_{0}=s_{0}$, then $s_{0}$ forms a fixed point of $T$, which completes the proof. Hence, we assume that $T s_{0} \neq s_{0}$. Starting from $s_{0}$, we construct inductively a sequence $\left\{s_{k}\right\}$, by:

$$
s_{k+1}=T^{n_{k}} s_{k} \text { for all } k \in \mathbb{N},
$$


where $n_{k}=n_{k}(s)$. Since:

$$
s_{1}=T^{n_{0}} s_{0} \text { and } s_{2}=T^{n_{1}} s_{1}=T^{n_{1}+n_{0}} s_{0},
$$

by induction, we deduce that:

$$
s_{k+1}=T^{n_{k}+\ldots+n_{1}+n_{0}} s_{0},
$$

which means that $\left\{s_{k}\right\}_{k} \geq 0$ is a subsequence of the orbit $\left\{T^{k} s_{0}: k \in \mathbb{N}\right\}$. Furthermore, we observe that:

$$
\begin{aligned}
s_{k+i} & =T^{n_{0}+n(1) \ldots+n_{k}+n_{k+1}+\ldots+n_{k+i-1}} s_{0} \\
& =T^{n_{k}+n_{k+1}+\ldots+n_{k+i-1}}\left(T^{n_{0}+n_{1}+\ldots+n_{k-1}} s_{0}\right) \\
& =T^{n_{k}+n_{k+1}+\ldots+n_{k+i-1}} s_{k} .
\end{aligned}
$$

We shall prove that $\left\{s_{n}\right\}$ is right-Cauchy and left-Cauchy in $(\mathcal{M}, q)$. First of all, notice that from (20),

$$
\begin{aligned}
q\left(s_{k}, s_{k+1}\right) & =q\left(s_{k}, T^{n_{k}} s_{k}\right)=q\left(T^{n_{k-1}} s_{k-1}, T^{n_{k}}\left(T^{n_{k-1}} s_{k-1}\right)\right) \\
& =q\left(T^{n_{k-1}} s_{k-1}, T^{n_{k-1}}\left(T^{n_{k}} s_{k-1}\right)\right),
\end{aligned}
$$

for any $k \in \mathbb{N}$ and replacing $s=s_{k-1}$ and $t=T^{n_{k}} s_{k-1}$ in (16), we get:

$$
\begin{aligned}
q\left(s_{k}, T^{n_{k}} s_{k}\right) & =q\left(T^{n_{k-1}} s_{k-1}, T^{n_{k-1}}\left(T^{n_{k}} s_{k-1}\right)\right) \\
& \leq \alpha\left(s_{k-1}, T^{n_{k}} s_{k-1}\right) q\left(T^{n_{k-1}} s_{k-1}, T^{n_{k-1}}\left(T^{n_{k}} s_{k-1}\right)\right) \\
& \leq \varphi\left(q\left(s_{k-1}, T^{n_{k}} s_{k-1}\right)\right) \\
& <q\left(s_{k-1}, T^{n_{k}} s_{k-1}\right) .
\end{aligned}
$$

Hence, since $\varphi$ is increasing, we obtain that:

$$
q\left(s_{k}, s_{k+1}\right)=q\left(s_{k}, T^{n_{k}} s_{k}\right) \leq \varphi^{k}\left(q\left(s_{0}, T^{n_{k}} s_{0}\right)\right) \leq \varphi^{k}\left(q\left(r_{1}\left(s_{0}\right)\right), \text { for all } k \in \mathbb{N} .\right.
$$

By using the triangular inequality and (24), for all $i \geq 1$, we get:

$$
\begin{aligned}
q\left(x_{k}, x_{k+l}\right) & \leq q\left(s_{k}, s_{k+1}\right)+q\left(s_{k+1}, s_{k+2}\right) \ldots+q\left(s_{k+l-2}, s_{k+l-1}\right)+q\left(s_{k+l-1}, s_{k+l}\right) \\
& \leq \sum_{j=k}^{k+l-1} \varphi^{j}\left(r_{1}\left(x_{0}\right)\right)<\sum_{j=k}^{\infty} \varphi^{j}\left(r_{1}\left(x_{0}\right)\right) .
\end{aligned}
$$

However, assuming that $T s_{0} \neq s_{0}$, we have $r_{1}\left(s_{0}\right) \geq q\left(s_{0}, T^{n_{k}} s_{0}\right)>0$, and by $(\Sigma)$, the series $\sum_{j=0}^{\infty} \varphi^{j}\left(r_{1}\left(s_{0}\right)\right)<\infty$ is convergent and then $\sum_{j=k}^{k+l-1} \varphi^{j}\left(r_{1}\left(s_{0}\right)\right) \rightarrow 0$. Therefore, for an arbitrary $\varepsilon>0$, there exists $k_{0} \in \mathbb{N}$ such that $\sum_{l=k}^{\infty} \varphi^{l}\left(r_{1}\left(s_{0}\right)\right)<\varepsilon$ for all $k \geq k_{0}$, and from (25),

$$
q\left(s_{k}, s_{k+l}\right)<\varepsilon,
$$

which ensures that $\left\{x_{k}\right\}$ is a right-Cauchy sequence. On account of Lemma 1 , we derive that $\left\{x_{k}\right\}$ is a left-Cauchy sequence in $(\mathcal{M}, q)$. Therefore, it is Cauchy in the complete quasi-metric space $(\mathcal{M}, q)$. It yields that there exists $u \in \mathcal{M}$ such that:

$$
\lim _{k \rightarrow \infty} q\left(s_{k}, u\right)=\lim _{k \rightarrow \infty} q\left(u, s_{k}\right)=0 .
$$

For the rest of the proof, we consider that $\left\{s_{k}\right\}$ is the sequence defined above and $u$ is the limit of this sequence.

Since $T$ is continuous, by using the property $(q 1)$ we derive that:

$$
\lim _{k \rightarrow \infty} q\left(s_{k}, T u\right)=\lim _{n \rightarrow \infty} q\left(T s_{k-1}, T u\right)=0,
$$


and:

$$
\lim _{n \rightarrow \infty} q\left(T u, s_{k}\right)=\lim _{n \rightarrow \infty} q\left(T u, T s_{k-1}\right)=0 .
$$

Thus, we have:

$$
\lim _{k \rightarrow \infty} q\left(s_{k}, T u\right)=\lim _{n \rightarrow \infty} q\left(T u, s_{k}\right)=0 .
$$

Keeping (26) and (29) in mind together with the uniqueness of a limit, we conclude that $u=T u$, that is $u$ is a fixed point of $T$.

In the following theorem, we remove the assumption of the continuity of the mapping $T$.

Theorem 5. Let $(\mathcal{M}, q)$ be a complete $\Delta$-symmetric quasi-metric space, T be a self-mapping and $\alpha: \mathcal{M} \times \mathcal{M} \rightarrow$ $\mathbb{R}_{0}^{+}, \zeta \in \mathcal{S}, \varphi \in \Psi$. Suppose that for every $x \in \mathcal{M}$, there is a positive integer $n=n(s)$ such that the inequality:

$$
\zeta\left(\alpha(s, t) q\left(T^{n(s)} s, T^{n(s)} t\right), \varphi(q(s, t))\right) \geq 0,
$$

is fulfilled for all $t \in \mathcal{M}$. Suppose also that:

(i) $T$ is a triangular $\alpha$-orbital admissible;

(ii) there exists $s_{0} \in \mathcal{M}$ such that $\alpha\left(s_{0}, T s_{0}\right) \geq 1$ and $\alpha\left(T s_{0}, s_{0}\right) \geq 1$;

(iii) $\mathcal{M}$ is $\alpha$-regular.

Then, $T$ has a fixed point $u \in \mathcal{M}$. Furthermore:

(a) for each $x \in \mathcal{M}, \lim _{m \rightarrow \infty} T^{m} s=u$;

(b) the mapping $T^{n(u)}$ is continuous at $u$.

Proof. As in Theorem 4, we can construct an iterative sequence $\left\{s_{n}\right\}$ that converges to a point $u \in \mathcal{M}$, which means that:

$$
\lim _{k \rightarrow \infty} q\left(s_{k}, u\right)=\lim _{k \rightarrow \infty} q\left(u, s_{k}\right)=0 .
$$

We claim, under the assumption that $\mathcal{M}$ is $\alpha$-regular, that $u$ is a fixed point of $T^{n}(u)$, that is $T^{n}(u) u=u$. First of all, replacing in (30) $s=s_{k-1}$ and $t=T^{n(u)} s_{k-1}$, we have for $n_{k-1}=n\left(s_{k-1}\right)$ :

$$
\zeta\left(\alpha\left(s_{k-1}, T^{n(u)} s_{k-1}\right) q\left(T^{n_{k-1}} s_{k-1}, T^{n_{k-1}}\left(T^{n(u)} s_{k-1}\right)\right), \varphi\left(q\left(s_{k-1}, T^{n(u)} s_{k-1}\right)\right)\right) \geq 0,
$$

or, keeping in mind $\left(\zeta_{1}\right)$,

$$
\begin{aligned}
q\left(s_{k}, T^{n(u)} s_{k}\right) & =q\left(T^{n_{k-1}} s_{k-1}, T^{n_{k-1}}\left(T^{n(u)} s_{k-1}\right)\right. \\
& \leq \alpha\left(s_{k-1}, T^{n(u)} s_{k-1}\right) q\left(T^{n_{k-1}} s_{k-1}, T^{n_{k-1}}\left(T^{n(u)} s_{k-1}\right)\right. \\
& \leq \varphi\left(q\left(s_{k-1}, T^{n(u)} s_{k-1}\right)\right. \\
& <q\left(s_{k-1}, T^{n(u)} s_{k-1}\right) .
\end{aligned}
$$

Taking into account that $\varphi$ is monotone increasing, we can write the chain of inequalities:

$$
\begin{aligned}
q\left(s_{k}, T^{n(u)} s_{k}\right) & \leq \varphi\left(q\left(s_{k-1}, T^{n(u)} s_{k-1}\right)\right. \\
& \leq \varphi^{2}\left(q\left(s_{k-2}, T^{n(u)} s_{k-2}\right)\right. \\
& \leq \ldots \leq \varphi^{k}\left(q\left(s_{0}, T^{n(u)} s_{0}\right) \leq \varphi^{k}\left(r_{1}\left(s_{0}\right)\right) .\right.
\end{aligned}
$$

Note that $\varphi \in \Psi$ is a $c$-comparison function, and hence, it satisfies $(\Omega)$ condition. Thus, we get that:

$$
\lim _{k \rightarrow \infty} q\left(s_{k}, T^{n(u)} s_{k}\right)=0
$$


Furthermore, from (35) and Lemma 1, we have:

$$
\lim _{k \rightarrow \infty} q\left(T^{n(u)} s_{k}, s_{k}\right)=0
$$

By contradiction, we assume that $T^{n(u)} u \neq u$, and let $\varepsilon_{1}=q\left(u, T^{n(u)} u\right)>0$ and $\varepsilon_{2}=$ $q\left(T^{n(u)} u, u\right)>0$. Without loss of generality, we suppose that $\varepsilon_{1} \leq \varepsilon_{2}$.

Again, since $\varphi \in \Psi,(\Gamma)$ holds, which means that:

$$
\varphi\left(\varepsilon_{1}\right)<\varepsilon_{1} .
$$

From (31) and respectively (36), there exists $k_{0} \in \mathbb{N}$ such that for any $k \geq k_{0}$, there hold:

$$
q\left(s_{k}, u\right)<\frac{\varepsilon_{1}}{3}, q\left(u, s_{k}\right)<\frac{\varepsilon_{1}}{3} \text { and } q\left(T^{n(u)} s_{k}, s_{k}\right)<\frac{\varepsilon_{1}}{3} .
$$

If $\mathcal{M}$ is regular with respect to $\alpha$, then there exists a subsequence $\left\{s_{k}\right\}$ of $\left\{s_{n}\right\}$ such that $\alpha\left(u, s_{k}\right) \geq 1$ for all $k$ and using the triangle inequality, we obtain:

$$
\begin{aligned}
\varepsilon_{2} & =q\left(T^{n(u)} u, u\right) \leq q\left(T^{n(u)} u, T^{n(u)} s_{k}\right)+q\left(T^{n(u)} s_{s_{k}} s_{k}\right)+q\left(s_{n_{k}}, u\right) \\
& \leq \alpha\left(u, s_{k}\right) q\left(T^{n(u)} u, T^{n(u)} s_{k}\right)+\frac{\varepsilon_{1}}{3}+\frac{\varepsilon_{1}}{3} \\
& <\varphi\left(q\left(u, s_{k}\right)+\frac{\varepsilon_{1}}{3}+\frac{\varepsilon_{1}}{3}\right. \\
& <\frac{\varepsilon_{1}}{3}+\frac{\varepsilon_{1}}{3}+\frac{\varepsilon_{1}}{3}=\varepsilon_{1},
\end{aligned}
$$

which is a contradiction. As a consequence, $T^{n(u)} u=u$.

Let us show now that uis the fixed point for $T$. Since $(\mathcal{M}, q)$ is $\alpha$-regular, using the triangle inequality, we have:

$$
\begin{aligned}
q(T u, u) & \left.=q\left(T\left(T^{n(u)} u\right), u\right)=q\left(T^{n(u)+1} u\right), u\right) \\
& \left.\left.\leq q\left(T^{n(u)+1} u\right), T^{n(u)+1} s_{k}\right)\right)+q\left(T^{n(u)+1} s_{s_{k}}, s_{k}\right)+q\left(s_{k}, u\right) \\
& \left.\left.\leq \alpha\left(u, s_{k}\right) q\left(T^{n(u)+1} u\right), T^{n(u)+1} s_{k}\right)\right)+q\left(T^{n(u)+1} s_{k}, s_{k}\right)+q\left(s_{k}, u\right) \\
& \leq \varphi\left(q\left(u, s_{k}\right)\right)+q\left(T^{n(u)+1} s_{s_{k}}, s_{k}\right)+q\left(s_{k}, u\right) \\
& <q\left(u, s_{k}\right)+q\left(T^{n(u)+1} s_{k}, s_{k}\right)+q\left(s_{k}, u\right) .
\end{aligned}
$$

Letting $k \rightarrow \infty$, together with (31) and (35), we obtain:

$$
q(T u, u) \leq 0
$$

Hence, $q(T u, u)=0$. Moreover, since the space is $\Delta$-symmetric, $q(u, T u) \leq M q(T u, u)=0$, then $q(u, T u)=q(T u, u)=0$, so $u$ is a fixed point of $T$.

We are demonstrating now (a). To begin, we claim that $\lim _{k \rightarrow \infty} q\left(u, T^{k} s\right)=0$, for each $x \in \mathcal{M}$. For this purpose, let $s \in \mathcal{M}$ be fixed, and let $p \in\{0,1, \ldots, n(u)-1\}$ be arbitrary. For all $k \geq 1$,

$$
\begin{aligned}
q\left(u, T^{\left.k n(u)+p_{S}\right)}\right. & =q\left(T^{n(u)} u, T^{n(u)}\left(T^{\left.(k-1) n(u)+p_{S}\right)}\right.\right. \\
& \leq \alpha\left(u, T^{(k-1) n(u)+p_{S}}\right) q\left(T^{n(u)} u, T^{n(u)}\left(T^{\left.(k-1) n(u)+p_{S}\right)}\right.\right. \\
& \leq \varphi\left(q\left(u, T^{\left.(k-1) n(u)+p_{S}\right)}\right)<q\left(u, T^{\left.(k-1) n(u)+p_{S}\right)}\right.\right.
\end{aligned}
$$

Since $\varphi$ is monotone increasing, repeatedly applying the previous inequality, we obtain:

$$
q\left(u, T^{\left.k n(u)+p_{S}\right)} \leq \varphi^{k}\left(q\left(u, T^{p} s\right)\right) .\right.
$$


Since $p, u$ and $s$ are fixed, we have $\lim _{n \rightarrow \infty} \varphi^{k}\left(q\left(u, T^{p} s\right)\right)=0$, which implies that:

$$
\lim _{n \rightarrow \infty}\left(q\left(u, T^{k n(u)+p} s\right)\right)=0 .
$$

If we do $p \in\{0,1, \ldots, n(u)-1\}$, we conclude that $\lim _{k \rightarrow \infty} q\left(u, T^{k} s\right)=0$. Taking Lemma 1 into account, we get that $\lim _{k \rightarrow \infty} q\left(T^{k} s, u\right)=0$ and also $\lim _{m \rightarrow \infty} T^{m} s=u$.

Finally, we prove that the statement (b) holds. For this purpose, we will show that $q\left(u, T^{n(u)} t_{k}\right) \leq$ $q\left(u, t_{k}\right)$ where $\left\{t_{k}\right\}$ is an arbitrary sequence in $\mathcal{M}$ such that:

$$
\lim _{k \rightarrow \infty} q\left(t_{k}, u\right)=\lim _{k \rightarrow \infty} q\left(u, t_{k}\right)=0 .
$$

Assume, by contradiction, that there exists some $k \in \mathbb{N}$ such that:

$$
q\left(u, T^{n(u)} t_{k}\right)>q\left(u, t_{k}\right) .
$$

Replacing in (30), we have:

$$
\zeta\left(\alpha\left(u, t_{k}\right) q\left(T^{n(u)} u, T^{n(u)} t_{k}\right), \varphi\left(q\left(u, t_{k}\right)\right)\right) \geq 0 .
$$

Since $\zeta \in \mathcal{S}$ and using the $\alpha$-regularity of $\mathcal{M}$, we have:

$$
\begin{aligned}
q\left(u, t_{k}\right) & <q\left(u, T^{n(u)} t_{k}\right)=q\left(T^{n(u)} u, T^{n(u)} t_{k}\right) \leq \alpha\left(u, t_{k}\right) q\left(T^{n(u)} u, T^{n(u)} t_{k}\right) \\
& \leq \varphi\left(q\left(u, t_{k}\right)\right)<q\left(u, t_{k}\right)
\end{aligned}
$$

which is a contradiction. As a consequence, $\lim _{k \rightarrow \infty} q\left(u, T^{n(u)} t_{k}\right)=0$. Since $q\left(T^{n(u)} t_{k}, u\right) \leq M q\left(u, T^{n(u)} t_{k}\right)$ for all $k \in \mathbb{N}$, we get that $\lim _{k \rightarrow \infty} q\left(T^{n(u)} t_{k}, u\right)=0$, and therefore:

$$
\lim _{k \rightarrow \infty} T^{n(u)} t_{k}=u
$$

which means that $T^{n(u)}$ is continuous at $s=u$.

We notice that to guarantee the uniqueness, we need to add an additional condition.

Theorem 6. Adding the condition ( $U$ ): If $u$ is a fixed point of $T^{n(u)}$ for any $t \in \mathcal{M}, \alpha(u, t) \geq 1$ to the statement of Theorem 4, respectively 5, we obtain the uniqueness of the fixed point.

Proof. By Theorem 4, we know that $T^{n(u)}$ has at least one fixed point. We suppose that there exists $v \in \mathcal{M}$ such that $T^{n(u)} v=v \neq u=T^{n(u)} u$. Then, due the condition (U):

$$
0<q(u, v)=q\left(T^{n(u)} u, T^{n(u)} v\right) \leq \alpha(u, v) q\left(T^{n(u)} u, T^{n(u)} v\right) \leq \varphi(q(u, v))<q(u, v)
$$

which is a contradiction.

In this case, it is obvious that $T u=T\left(T^{n(u)} u\right)=T^{n(u)}(T u)$ which shows that $T u$ is a fixed point of $T$, and due to its uniqueness, we conclude that $T u=u$.

Example 2. Let $\mathcal{M}=[0,4], q: \mathcal{M} \times \mathcal{M} \rightarrow \mathbb{R}_{0}^{+}$, defined by:

$$
q(s, t)=\left\{\begin{aligned}
2 \cdot(s-t) & \text { if } s \geq t \\
(t-s) & \text { otherwise }
\end{aligned}\right.
$$


It easy to see that $(\mathcal{M}, q)$ is a complete two-symmetric quasi-metric space. Let $T: \mathcal{M} \rightarrow \mathcal{M}$,

$$
T(s)= \begin{cases}\frac{s}{2} & \text { if } s \in[0,1] \\ 3 & \text { if } s \in(1,2) \\ \frac{s}{4} & \text { if } s \in[2,4]\end{cases}
$$

and $\alpha: \mathcal{M} \times \mathcal{M} \rightarrow \mathbb{R}_{0}^{+}$,

$$
\alpha(s, t)= \begin{cases}2 & \text { if } s, t \in[0,1] \\ 1 & \text { if }(s, t) \in\{[0,1] \times[2,4]\} \cup\{[2,4] \times[0,1]\} \cup\left\{\left(\frac{1}{2}, \frac{3}{2}\right)\right\} \\ 0 & \text { otherwise }\end{cases}
$$

We shall prove that $T$ satisfies the conditions of Theorem 5 . If $s \in[0,1]$, then $T s, T^{2} s \in[0,1]$ and $\alpha(s, T s)=2 \geq 1 \Rightarrow \alpha\left(T s, T^{2} s\right)=2 \geq 1$. Furthermore, if $s \in[2,4]$, then $T s, T^{2} s \in[0,1]$ and $\alpha(s, T s)=1 \geq$ $1 \Rightarrow \alpha\left(T s, T^{2} s\right)=2 \geq 1$. Hence, $T$ is an $\alpha$-orbital mapping. For $\alpha(s, t) \geq 1$, we consider the following cases:

(a) If $s, t \in[0,1]$, then $T t \in[0,1], \alpha(t, T t)=2 \geq 1$ and $\alpha(s, T t)=2 \geq 1$;

(b) If $s \in[0,1], t \in[2,4]$, then $T t \in[0,1], \alpha(t, T t)=1 \geq 1$ and $\alpha(s, T t)=2 \geq 1$;

(c) If $s \in[2,4], t \in[0,1]$, then $T t \in[0,1], \alpha(t, T t)=2 \geq 1$ and $\alpha(s, T t)=1 \geq 1$;

(d) If $s=\frac{1}{2}, t=\frac{3}{2}$, since $T \frac{3}{2}=3, \alpha\left(\frac{3}{2}, 3\right)=0$.

Therefore, $T$ is a triangular $\alpha$-orbital admissible mapping, so the assumption (i) of Theorem 5 is satisfied.

Remark 1. We remark that, since $\alpha\left(3, \frac{1}{2}\right)=\alpha\left(\frac{1}{2}, \frac{3}{2}\right)=1$ and $\alpha\left(3, \frac{3}{2}\right)=0, T$ is not triangular $\alpha$-admissible mapping.

Further, Condition (ii) is satisfied, since $\alpha(0, T 0)=\alpha(0,0)=2$. Moreover, if $\left\{s_{n}\right\}$ is a sequence in $\mathcal{M}$ such that $\alpha\left(s_{n}, s_{n+1}\right) \geq 1$ for all $n \in \mathbb{N}$ and $\lim _{n \rightarrow \infty} s_{n}=s \in \mathcal{M}$, then $s=0$ and $\alpha\left(0, s_{n}\right)$ geq 1 for all $n$. Hence, $\mathcal{M}$ is $\alpha$-regular. Due to the manner in which we defined the function $\alpha$, the following cases are the interesting ones, letting, for example $\zeta(t, s)=\frac{s}{2}-t$ and $\varphi(t)=\frac{t}{3}$.

(1) For $s \in[0,1], t \in[0,1]$, we have $T^{s}=\frac{s}{2^{n}}$ and $T^{n} t=\frac{t}{2^{n}}$. If $s \geq t$, then $q(s, t)=2(s-t)$ and $q\left(T^{n} s, T^{n} t\right)=2 \frac{s-t}{2^{n}}$. Thus, (14) becomes:

$$
\alpha(s, t) q\left(T^{n} s, T^{n} t\right)=2 \cdot 2 \frac{s-t}{2^{n}} \leq \frac{1}{2} \frac{2(s-t)}{3}=\frac{1}{2} \varphi(q(s, t))
$$

which holds for any $n \in\{1,2, \ldots\}$. If $s<t$, then $q(s, t)=t-s$ and $q\left(T^{n} s, T^{n} t\right)=\frac{t-s}{2^{n}}$. In this case,

$$
\alpha(s, t) q\left(T^{n} s, T^{n} t\right)=2 \cdot \frac{t-s}{2^{n}} \leq \frac{1}{2} \cdot \frac{(t-s)}{3}=\frac{1}{2} \varphi(q(s, t)),
$$

holds also for any $n \in\{1,2, \ldots\}$.

(2) For $s \in[0,1], t \in[2,4]$, we have $s<t, T^{s}=\frac{s}{2^{n}}, T^{n} t=\frac{t}{4 \cdot 2^{n-1}}=\frac{t}{2^{n+1}}$ and $T^{n} s \leq T^{n} t$. Hence,

$$
\alpha(s, t) q\left(T^{n} s, T^{n} t\right)=2 \cdot \frac{t-2 s}{2^{n+1}} \leq \frac{1}{2} \frac{(t-s)}{3}=\frac{1}{2} \varphi(q(s, t)),
$$

(3) For $s \in[2,4], t \in[0,1]$, we have $s>t, T^{n} s=\frac{s}{2^{n+1}}, T^{n} t=\frac{t}{2^{n}}$ and $T^{n} s \geq T^{n} t$. Hence,

$$
\alpha(s, t) q\left(T^{n} s, T^{n} t\right)=2 \cdot \frac{2(s-2 t)}{2^{n+1}} \leq 2 \cdot \frac{1}{2} \frac{(s-t)}{3}=\frac{1}{2} \varphi(q(s, t)),
$$


(4) For $s=\frac{1}{2}$ and $t=\frac{3}{2}$, we have $T^{n} \frac{1}{2}=\frac{1}{2^{n+1}}, T^{n} \frac{3}{2}=T^{n-1}\left(T_{\frac{3}{2}}\right)=T^{n-1} 3=T^{n-2}(T 3)=T^{n-2} \frac{3}{4}=$ $\frac{3}{4 \cdot 2^{n-2}}=\frac{3}{2^{n}}$, so $T^{n} \frac{1}{2}<T^{n} \frac{3}{2}$. Hence,

$$
\alpha\left(\frac{1}{2}, \frac{3}{2}\right) q\left(T^{n} \frac{1}{2}, T^{n} \frac{3}{2}\right)=\left(\frac{3}{2^{n}}-\frac{1}{2^{n}+1}\right)=\frac{5}{2^{n+1}} \leq \frac{1}{6}=\frac{1}{2} \frac{\left(\frac{3}{2}-\frac{1}{2}\right)}{3}=\frac{1}{2} \varphi(q(s, t)),
$$

Therefore, for any $s \in \mathcal{M}$, there exists $n(s) \in \mathbb{N}$, for example $n(s)=4$, such that for every $t \in \mathcal{M}$, all assumptions of Theorem 4 are satisfied. Then, $T$ has a (unique) fixed point $u=0$. On the other hand, $q\left(T \frac{1}{2}, T \frac{3}{2}\right)=q\left(\frac{1}{4}, 3\right)=3-\frac{1}{4}=\frac{11}{4}, q\left(\frac{1}{2}, \frac{3}{2}\right)=\frac{3}{2}-\frac{1}{2}=1$ and $q\left(T \frac{1}{2}, T \frac{3}{2}\right) \leq k \cdot q\left(\frac{1}{2}, \frac{3}{2}\right)$ imply $k>1$. Hence $T$ is not a contraction. Moreover, we cannot find the functions $\zeta \in \mathcal{S}, \varphi \in \phi$ such that:

$$
\zeta\left(\alpha\left(\frac{1}{2}, \frac{3}{2}\right) q\left(T \frac{1}{2}, T \frac{3}{2}\right), \varphi\left(q\left(\frac{1}{2}, \frac{3}{2}\right)\right)\right) \geq 0 .
$$

This shows that Theorem 5 is indeed a generalization of known results.

Theorem 7. Let $(\mathcal{M}, q)$ be a complete $\Delta$-symmetric quasi-metric space, a self-mapping $T$ and a map $\alpha$ : $\mathcal{M} \times \mathcal{M} \rightarrow \mathbb{R}_{0}^{+}$. Suppose that there exist $\zeta \in \mathcal{S}, \varphi \in \phi$ such that for every $s \in \mathcal{M}$, there is a positive integer $n=n(s)$ such that for all $t \in \mathcal{M}$ :

$$
\zeta(\alpha(s, t) q(s, t), \varphi(S(s, t))) \geq 0,
$$

for each $s, t \in \mathcal{M}$, where:

$$
S(s, t)=\max \left\{q(s, t), q\left(s, T^{n(s)} s\right), q\left(s, T^{n(s)} t\right)\right\} .
$$

Assume that:

(i) T forms a triangular $\alpha$-orbital admissible;

(ii) there is $s_{0} \in \mathcal{M}$ such that $\alpha\left(s_{0}, T s_{0}\right) \geq 1$ and $\alpha\left(T s_{0}, s_{0}\right) \geq 1$;

(iii)a either, $\mathcal{M}$ is $\alpha$-regular,

(iii)b or, $T$ is continuous.

Then, $T$ has a fixed point $u \in \mathcal{M}$. Furthermore, for each $s \in \mathcal{M}, \lim _{m \rightarrow \infty} T^{m} s=u$.

Proof. We remark firstly that if $s \in \mathcal{M}$, then there exist $n=n(s)$ such that for all $y \in \mathcal{M}$ :

$$
\zeta(\alpha(s, t) q(s, t), \varphi(S(s, t))) \geq 0,
$$

or, with $(\zeta 1)$ in mind,

$$
\begin{aligned}
0 & \leq \zeta(\alpha(s, t)(q(s, t), \varphi(S(s, t))) \\
& <\varphi(S(s, t))-\alpha(s, t) q(s, t) .
\end{aligned}
$$

Furthermore,

$$
\alpha(s, t) q(s, t) \leq \varphi(S(s, t)) .
$$

The functions $r_{1}(s)$ and $r_{2}(s)$, defined by (17), are finite, which is observed by following step by step the lines in the proof of Theorem 4 . By substituting $s$ by $T^{n(s)}$ and $y$ by $T^{(k-1) n(x)+p_{S}}$ in (45) and taking $(\Gamma)$ into account, we have:

$$
\alpha\left(x, T^{(k-1) n(s)+p} s\right) q\left(s, T^{(k-1) n(s)+p} s\right) \leq \varphi\left(S\left(s, T^{(k-1) n(s)+p} s\right)\right),
$$

where: 


$$
\begin{aligned}
S\left(s, T^{\left.(k-1) n(s)+p_{s}\right)}\right. & =\max \left\{q\left(s, T^{(k-1) n(s)+p_{S}}\right), q\left(s, T^{n(s)} s\right), q\left(s, T^{n(s)}\left(T^{(k-1) n(s)+p_{S}}\right)\right)\right\} \\
& =\max \left\{q\left(s, T^{(k-1) n(s)+p_{S}}\right), q\left(s, T^{n(s)} s\right), q\left(s, T^{\left.k n(s)+p_{S}\right)}\right\}\right.
\end{aligned}
$$

Denoting by $a_{k}=q\left(s, T^{k n(s)+p_{s}}\right)$ for $k \in \mathbb{N}$, from the triangle inequality, we have:

$$
\begin{aligned}
a_{k}=q\left(s, T^{\left.k n(s)+p_{s}\right)}\right. & \leq q\left(s, T^{n(s)} s\right)+q\left(T^{n(s)} s, T^{\left.k n(s)+p_{s}\right)}\right. \\
& =q\left(s, T^{n(s)} x\right)+q\left(T^{n(s)} s, T^{n(s)}\left(T^{(k-1) n(s)+p_{s}}\right) .\right.
\end{aligned}
$$

and combining with (46), we get, for any $k \in \mathbb{N}$ :

$$
\begin{aligned}
& a_{k} \leq q\left(s, T^{n(s)} s\right)+q\left(T^{n(s)} s, T^{n(s)}\left(T^{\left.(k-1) n(s)+p_{s}\right)}\right)\right. \\
& \leq q\left(s, T^{n(s)} s\right)+\alpha\left(s, T^{(k-1) n(s)+p_{s}}\right) q\left(T^{n(s)} s, T^{n(s)}\left(T^{(k-1) n(s)+p_{s}}\right)\right) \\
& \leq q\left(s, T^{n(s)} s\right)+\varphi\left(S\left(s, T^{\left.(k-1) n(s)+p_{s}\right)}\right)\right. \\
& =q\left(s, T^{n(s)} s\right)+\varphi\left(\max \left\{q\left(s, T^{(k-1) n(s)+p_{s}}\right), q\left(s, T^{n(s)} s\right), q\left(s, T^{k n(s)+p_{s}}\right)\right\}\right) \\
& =q\left(s, T^{n(s)} s\right)+\varphi\left(\max \left\{a_{k-1}, q\left(s, T^{n(s)} s\right), a_{k}\right\}\right) \text {. }
\end{aligned}
$$

Following the reasoning in the previous theorem, notice that we can find $c \in(0, \infty)$, with $c>l=\max \left\{a_{0}, q\left(s, T^{n}(s) s\right)\right\} \geq a_{0}$ such that $t-\varphi(t)>l$ for all $t \in \mathbb{R}_{0}^{+}$. We shall prove that $a_{k}<c$ for all $k \in \mathbb{N}$. For this, we assume, on the contrary, namely, that there exists a positive integer $i$ such that $a_{i-1}<c \leq a_{i}$. We have then:

$$
a_{i} \leq l+\varphi\left(\max \left\{a_{i-1}, l, a_{i}\right\}\right) \leq l+\varphi\left(a_{i}\right)
$$

or $a_{i}-\varphi\left(a_{i}\right) \leq l$, which is a contradiction. Therefore, the set $\left\{q\left(s, T^{n} s\right): n \in \mathbb{N}\right\}$ is bounded and $r_{1}(s)<\infty$. Since the space $(\mathcal{M}, q)$ is $\Delta$-symmetric, we have for all $s \in \mathcal{M}$ and all $k \in \mathbb{N}$ that:

$$
q\left(T^{n} s, s\right) \leq M q\left(s, T^{n} s\right) \leq M r_{1}(s),
$$

which shows that the set $\left\{q\left(T^{n} s, s\right): n \in \mathbb{N}\right\}$ is also bounded. Hence, $r_{2}(s)<\infty$.

Let $s_{0} \in \mathcal{M}$ be arbitrary. We will show now that the sequence $\left\{s_{i}\right\}$ constructed inductively by:

$$
\begin{aligned}
& m_{0}=n\left(s_{0}\right), s_{1}=T^{m_{0}} s_{0} \\
& m_{i}=n\left(s_{i}\right), s_{i+1}=T^{m_{i}} s_{i}
\end{aligned}
$$

is a Cauchy sequence. By this construction of sequence $\left\{s_{i}\right\}$, it follows that for any $i \in \mathbb{N}$ and $l \geq 1$,

$$
\begin{aligned}
s_{i+l} & =T^{m_{i+l-1}} s_{i+l-1}=T m_{i+l-1}+\ldots+m_{i+1}+m_{i} x_{i} \\
& =T^{m_{i+l-1}+\ldots+m_{i+1}+m_{i}+m_{i-1} \ldots+m_{1}+m_{0}} s_{0} .
\end{aligned}
$$

If we denote by $s_{0}=m_{i+l-1}+\ldots+m_{i+1}+m_{i}$, using the condition (42) or, equivalently, (45), for $s=s_{i-1}$ and $t=T^{p_{0}} t_{i-1}$, we have:

$$
\begin{aligned}
q\left(s_{i}, s_{i+l}\right) & =q\left(x_{i}, T^{p_{0}} s_{i}\right) \leq \alpha\left(s_{i-1}, T^{p_{0}} s_{i-1}\right) q\left(T^{m_{i-1}} s_{i-1}, T^{m_{i-1}}\left(T^{p_{0}} s_{i-1}\right)\right. \\
& \leq \varphi\left(S\left(s_{i-1}, T^{p_{0}} s_{i-1}\right)\right)
\end{aligned}
$$

where:

$$
\left.S\left(s_{i-1}, T^{p_{0}} s_{i-1}\right)=\max \left\{q\left(s_{i-1}, T^{p_{0}} s_{i-1}\right), T\left(s_{i-1}, T^{m_{i-1}} s_{i-1}\right), q\left(s_{i-1}, T^{p_{0}+m_{i-1}} s_{i-1}\right)\right)\right\} .
$$


Let us now have a positive integer $p_{1} \in\left\{p_{0}, m_{i-1}, p_{0}+m_{i-1}\right\}$ such that $S\left(s_{i-1}, T^{p_{0}} s_{i-1}\right)=$ $q\left(s_{i-1}, T^{p_{1}} s_{i-1}\right)$. Replace in (50):

$$
q\left(s_{i}, T^{p_{0}} s_{i}\right) \leq \varphi\left(q\left(s_{i-1}, T^{p_{1}} s_{i-1}\right)\right) .
$$

Hence, $\varphi$ is monotone increasing, and we obtain that:

$$
\begin{aligned}
q\left(s_{i}, s_{i+l}\right) & =q\left(s_{i}, T^{p_{0}} s_{i}\right) \leq \varphi\left(q\left(s_{i-1}, T^{p_{1}} s_{i-1}\right)\right) \leq \varphi^{2}\left(q\left(s_{i-2}, T^{p_{2}} s_{i-2}\right)\right) \\
& \leq \ldots \leq \varphi^{i}\left(q\left(s_{0}, T^{p_{i}} s_{0}\right)\right) \leq \varphi^{i}\left(r_{1}\left(s_{0}\right)\right),
\end{aligned}
$$

for $p_{0}, p_{1}, \ldots, p_{i} \in \mathbb{N}$. However, $\varphi$ is a $c$-comparison function, and from $(\Omega)$, there exist $\varepsilon>0$ such that, $\varphi^{i}\left(r_{1}\left(s_{0}\right)\right)<0$ for any $i \in \mathbb{N}$. Hence,

$$
q\left(s_{i}, s_{i+l}\right)<\varepsilon
$$

for arbitrary $i \in \mathbb{N}$ and $l \geq 1$. We conclude that $\left\{l_{i}\right\}$ is a right-Cauchy sequence on $(\mathcal{M}, q)$. Since $(\mathcal{M}, q)$ is supposed to be $\Delta$-symmetric, then, from Lemma 1, it is a Cauchy sequence. As the space is complete, there exists $u \in \mathcal{M}$ such that $s_{n} \rightarrow u$, which means that:

$$
\lim _{i \rightarrow \infty} q\left(s_{i}, u\right)=\lim _{i \rightarrow \infty} q\left(u, s_{i}\right)=0 .
$$

If we use the hypothesis that the map $T$ is continuous, we obtain:

$$
u=\lim _{i \rightarrow \infty} s_{i+1}=\lim _{i \rightarrow \infty} T s_{i}=T u
$$

that is, $u$ is a fixed point of $T$.

We want to show now that $T^{n(u)} u=u$, that is $u$ is a fixed point of $T^{n(u)}$, under the assumption that $\mathcal{M}$ is $\alpha$-regular. First, we show that:

$$
\lim _{i \rightarrow \infty} q\left(s_{i}, T^{n(u)} s_{i}\right)=0
$$

and:

$$
\lim _{i \rightarrow \infty} q\left(T^{n(u)} s_{i}, s_{i}\right)=0
$$

By proceeding as above, from (45), we get:

$$
\begin{aligned}
q\left(s_{i}, T^{n(u) s_{i}}\right) & =q\left(T^{m_{i-1}} s_{i-1}, T^{m_{i-1}}\left(T^{n(u)} s_{i-1}\right)\right. \\
& \leq \alpha\left(s_{i-1}, T^{n(u)} s_{i-1}\right) q\left(T^{m_{i-1}} s_{i-1}, T^{m_{i-1}}\left(T^{n(u)} s_{i-1}\right)\right. \\
& \leq \varphi\left(S\left(s_{i-1}, T^{n(u)} s_{i-1}\right)\right) \\
& \left.=\varphi\left(\max \left\{q\left(s_{i-1}, T^{n(u)} s_{i-1}\right), T\left(s_{i-1}, T^{m_{i-1}} s_{i-1}\right), q\left(s_{i-1}, T^{n(u)+m_{i-1}} s_{i-1}\right)\right)\right\}\right) \\
& =\varphi\left(q\left(s_{i-1}, T^{r_{i}} s_{i-1}\right)\right),
\end{aligned}
$$

where $r_{i} \in\left\{n(u), m_{i-1}, n(u)+m_{i-1}\right\}$ is an appropriate index such that $S\left(s_{i-1}, T^{n(u)} s_{i-1}\right)=$ $q\left(s_{i-1}, T^{r_{1}} s_{i-1}\right)$. Using the same arguments as previously given and taking into account that $\varphi$ is monotone increasing, we obtain that:

$$
q\left(s_{i}, T^{n(u)} s_{i}\right) \leq \varphi^{i}\left(q\left(s_{0}, T^{r_{i}} s_{0}\right) \rightarrow 0 .\right.
$$

Therefore, indeed, (54) holds. We remark than from Lemma 1, also (55) holds. Let $\varepsilon>0$ be arbitrary chosen. Then, there exists $i_{0} \in \mathbb{N}$ such that for any $i \geq i_{0}$, the following hold:

$$
q\left(s_{i}, u\right)<\frac{\varepsilon}{3}, q\left(u, s_{i}\right)<\frac{\varepsilon}{3}, q\left(T^{n(u)} s_{i}, s_{i}\right)<\frac{\varepsilon}{3} .
$$


Consequently, since $(\mathcal{M}, q)$ is $\alpha$-regular, and $\varphi \in \Psi$, we have:

$$
\begin{aligned}
q\left(T^{n(u)} u, u\right) & \leq q\left(T^{n(u)} u, T^{n(u)} s_{i}\right)+q\left(T^{n(u)} s_{i}, s_{i}\right)+q\left(s_{i}, u\right) \\
& \leq \alpha\left(u, s_{i}\right) q\left(T^{n(u)} u, T^{n(u)} s_{i}\right)+q\left(T^{n(u)} s_{i}, s_{i}\right)+q\left(s_{i}, u\right) \\
& \leq \varphi\left(S\left(u, s_{i}\right)\right)+q\left(T^{n(u)} s_{i}, s_{i}\right)+q\left(s_{i}, u\right) \\
& \leq \varphi\left(\frac{\varepsilon}{3}\right)+\frac{\varepsilon}{3}+\frac{\varepsilon}{3} \\
& \leq \frac{\varepsilon}{3}+\frac{\varepsilon}{3}+\frac{\varepsilon}{3}=\varepsilon .
\end{aligned}
$$

Thus, it follows that $T^{n(u)} u=u$. We claim now that $T^{n} s \rightarrow u$, for each $x \in \mathcal{M}$, that is $q\left(T^{n} s, u\right)=$ $q\left(u, T^{n} s\right)=0$. For $s \in \mathcal{M}$ fixed and $p \in\{0,1,2, \ldots, n(u)-1\}$,

$$
\begin{aligned}
q\left(u, T^{\left.k n(u)+p_{S}\right)}\right. & =q\left(T^{n(u)} u, T^{n(u)}\left(T^{\left.\left.(k-1) n(u)+p_{S}\right)\right)}\right.\right. \\
& \leq \alpha\left(u, T^{(k-1) n(u)+p_{u}}\right) q\left(T^{n(u)} u, T^{n(u)}\left(T^{\left.(k-1) n(u)+p_{S}\right)}\right)\right. \\
& \leq \varphi\left(S \left(u, T^{\left.\left.(k-1) n(u)+p_{S}\right)\right)}\right.\right. \\
& =\varphi\left(\max \left\{q\left(u, T^{(k-1) n(u)+p_{S}}\right), q\left(u, T^{n(u)} u\right), q\left(u, T^{n(u)}\left(T^{(k-1) n(u)+p_{S}}\right)\right)\right\}\right) \\
& =\varphi\left(\max \left\{q\left(u, T^{(k-1) n(u)+p_{S}}\right), q\left(u, T^{\left.k n(u)+p_{S}\right)}\right\}\right) .\right.
\end{aligned}
$$

$$
\begin{aligned}
& \text { If } \max \left\{q\left(u, T^{(k-1) n(u)+p_{S}}\right), q\left(u, T^{k n(u)+p_{S}}\right)\right\}=q\left(u, T^{k n(u)+p_{S}}\right) \text {, then: } \\
& \qquad q\left(u, T^{k n(u)+p_{S}}\right) \leq \varphi\left(q\left(u, T^{k n(u)+p_{s}}\right)\right)<q\left(u, T^{k n(u)+p_{S}}\right)
\end{aligned}
$$

which is a contradiction. Hence, $q\left(u, T^{k n(u)+p_{S}}\right) \leq q\left(u, T^{\left.(k-1) n(u)+p_{S}\right)}\right.$. Since $\varphi \in \Psi, \varphi$ is a comparison function, we obtain:

$$
q\left(u, T^{k n(u)+p_{s}}\right) \leq \varphi\left(q\left(u, T^{(k-1) n(u)+p_{s}}\right)\right) \leq \ldots \leq \varphi^{k}\left(q\left(u, T^{p} s\right) \rightarrow 0,\right.
$$

where $u, s \in \mathcal{M}$ and $p \geq 1$ are fixed. Using Lemma 1 , we obtain that, for any $n \in \mathbb{N}$,

$$
\lim _{n \rightarrow \infty} T^{n} s=u
$$

Next, we shall show the uniqueness of the obtained fixed point of $T$, defined in Theorem 7 . In order to prove the uniqueness of the fixed point, we propose the following condition:

(U) If $u$ is a fixed point of $T^{n(u)}$ for any $t \in \mathcal{M}, \alpha(u, t) \geq 1$.

Theorem 8. Besides all assumptions of Theorem 7 , suppose that the condition $(U)$ is satisfied. Then, $T$ has a unique fixed point.

Proof. The existence of a fixed point is observed by Theorem 7. For the proof of the uniqueness, we use the method of reductio ad absurdum. Let $u, v \in \mathcal{M}$ be two fixed points of $T^{n(u)}$ with $u \neq v$. Then, we have from hypotheses $(U)$ that $\alpha(u, v) \geq 1$ and:

$$
\begin{aligned}
q(u, v) & \leq \alpha(u, v) q\left(T^{n(u)} u, T^{n(u)} v\right) \leq \varphi(S(u, v)) \\
& \leq \varphi\left(\max \left\{q(u, v), q\left(u, T^{n(u)} u\right), q\left(u, T^{n(u)} v\right)\right\}\right) \\
& \leq \varphi(\max q(u, v), q(u, u))=\varphi(q(u, v))<q(u, v),
\end{aligned}
$$

which is a contradiction. Hence, $u$ is the unique fixed point for $T^{n(u)}$. Then, $T u=T\left(T^{n(u)} u\right)=$ $T^{n(u)}(T u)$ implies that $T u=u$, which shows that $u$ is the unique fixed point of $\mathrm{T}$. 
Example 3. Let $\mathcal{M}=\{0,1,2,3\}, q: \mathcal{M} \times \mathcal{M} \rightarrow \mathbb{R}_{0}^{+}$, defined by:

$$
\begin{aligned}
& q(1,2)=q(2,1)=2, q(3,2)=q(2,3)=2 \\
& q(2,0)=q(0,2)=3, q(1,3)=q(3,1)=1 \\
& q(1,0)=q(3,0)=2, q(0,1)=q(0,3)=1 \\
& q(0,0)=q(1,1)=q(2,2)=q(3,3)=0 .
\end{aligned}
$$

Then, $(\mathcal{M}, q)$ is a two-symmetric quasi-metric space. Let $T: \mathcal{M} \rightarrow \mathcal{M}, T 0=0, T 1=2, T 2=3, T 3=0$ and $\alpha: \mathcal{M} \times \mathcal{M} \rightarrow \mathbb{R}_{0}^{+}$be defined by $\alpha(s, t)=1$ if $(s, t) \in\{(0,0),(1,2),(1,3),(2,3),(1,0),(2,0)(3,0)\}$ and $\alpha(s, t)=0$ otherwise. Since:

$$
\begin{aligned}
& \alpha(0, T 0)=\alpha(0,0)=1 \Rightarrow \alpha\left(T 0, T^{2} 0\right)=\alpha(0,0)=1 \\
& \alpha(1, T 1)=\alpha(1,2)=1 \Rightarrow \alpha\left(T 1, T^{2} 1\right)=\alpha(2,3)=1 \\
& \alpha(2, T 2)=\alpha(2,3)=1 \Rightarrow \alpha\left(T 2, T^{2} 2\right)=\alpha(3,0)=1 \\
& \alpha(3, T 3)=\alpha(3,0)=1 \Rightarrow \alpha\left(T 3, T^{2} 3\right)=\alpha(0,0)=1
\end{aligned}
$$

$T$ is $\alpha$-orbital admissible. Furthermore, it is easy to check that $T$ is triangular $\alpha$-orbital admissible, but is not triangular $\alpha$ admissible, since, for example,

$$
\alpha(2,3)=1 \text { and } \alpha(3,0)=1 \text { but } \alpha(2,0)=0<1 .
$$

Notice that, for $n \geq 4$, we have $T^{n} s=0$. Hence, for any $\zeta \in \mathcal{S}, \varphi \in \phi$ such that for every $s \in \mathcal{M}$, there is a positive integer $n=n(s)$ (for example, any $n \in\{5,6, \ldots\}$ ) such that for all $t \in \mathcal{M}$, all conditions of Theorem 7 are satisfied, and $s=0$ is the fixed point of $T$. We notice that $T$ is not a contraction, since for example, for $s=1$ and $t=3$, we have:

$$
q(T 1, T 3)=q(2,0)=3>k \cdot 1=k \cdot q(1,3)
$$

for all $k \in[0,1)$.

\section{Ulam Stability}

There is a strong relation between fixed point theory and Ulam stability; see, e.g., [36-41].

Definition 7. Let $(\mathcal{M}, q)$ be a $\Delta$-symmetric quasi-metric space and $T: \mathcal{M} \rightarrow \mathcal{M}$ be a mapping. The fixed point equation:

$$
s=T s, s \in \mathcal{M}
$$

is called generalized Ulam stable if for each $\varepsilon>0$ and $s \in \mathcal{M}$, there exists $n(s) \in\{1,2, \ldots\}$ such that for any $v \in \mathcal{M}$ satisfying the inequality:

$$
q\left(T^{n(v)} v, v\right) \leq \varepsilon
$$

there exists an increasing function $\beta: \mathbb{R}_{0}^{+} \rightarrow \mathbb{R}_{0}^{+}$continuous at zero, with $\beta(0)=0$ and $u \in \mathcal{M}$ a solution of Equation (57) such that:

$$
q(u, v) \leq \beta(\varepsilon) \text { and } q(v, u) \leq \beta(\varepsilon) .
$$

Remark 2. If $\beta(t)=c t$ for all $t \geq 0$, where $c>0$, the fixed point Equation (57) is said to be Ulam stable.

Theorem 9. Let $(\mathcal{M}, q)$ be a complete $\Delta$-symmetric quasi-metric space. Let the function $\beta: \mathbb{R}_{0}^{+} \rightarrow \mathbb{R}_{0}^{+}$, defined by $\beta(t):=t-\varphi(t)$, with $\varphi \in \Psi$. Suppose that the hypothesis of Theorem 6 is satisfied. Then, the fixed point Equation (57) is generalized Ulam stable. 
Proof. From Theorem 6, there exists a unique $u \in \mathcal{M}$ such that $T u=u$, which means that $u$ is a solution of fixed point Equation (57). Let $v \in \mathcal{M}$. There exist $n(v) \in\{1,2, \ldots\}$ such that (58) holds. Using condition (14), for $s=u$ and $t=v$, we get:

$$
\zeta\left(\alpha(u, v) q\left(T^{n(v)} u, T^{n(v)} v\right), \varphi(q(u, v))\right) \geq 0
$$

Keeping in mind the properties of $\zeta, \varphi$, the condition $(U)$ imposed on the alpha function and using the triangle inequality, we obtain:

$$
\begin{aligned}
q(u, v) & \leq q\left(u, T^{n(v)} u\right)+q\left(T^{n(v)} u, T^{n(v)} v\right)+q\left(T^{n(v)} v, v\right) \\
& =q(u, u)+q\left(T^{n(v)} u, T^{n(v)} v\right)+q\left(T^{n(v)} v, v\right) \\
& \leq \alpha(u, v) q\left(T^{n(v)} u, T^{n(v)} v\right)+\varepsilon \\
& \leq \varphi(q(u, v))+\varepsilon .
\end{aligned}
$$

Taking into account the definition of the function $\beta$, we have:

$$
q(u, v)-\varphi(q(u, v))=\beta(q(u, v)) \leq \varepsilon
$$

or,

$$
q(u, v) \leq \beta^{-1}(\varepsilon)
$$

and since $\beta$ is continuous, strictly increasing, $\beta^{-1}$ is also increasing, continuous with $\beta^{-1}(0)=0$ On the other hand, the space $(\mathcal{M}, q)$ is $\Delta$-symmetric, so that, for a given positive real number $M>0$ :

$$
q(v, u) \leq M \cdot q(u, v) \leq M \beta^{-1}(\varepsilon)
$$

for all $u, v \in \mathcal{M}$. Hence, the fixed point Equation (57) is generalized Ulam stable.

\section{Conclusions}

In this paper, we obtain fixed point theorems in quasi-metric spaces by using simulations functions. Notice that the class of simulation functions is quite rich; see, e.g., [6,7,18-20,22]. Accordingly, for each simulation function, we find not only the existing results in the literature, but also some new results; in particular, by letting simulation function $\zeta(t, s)=q s-t, q \in(0,1)$ in Theorem 4 .

On the other hand, we have used an auxiliary function $\alpha$ in simulation functions that unifies the results in standard (quasi-)metric and results in a partially ordered (quasi-)metric and also the frame of the cyclic mappings. As was shown in several papers (see, e.g., [26,28,35]), by taking the auxiliary function $\alpha$ in a proper way, we shall get analogous results in a partially ordered structure and in the setting of cyclic maps. Regarding these aspects and several possible combinations of them, one can get a long list of corollaries of the results of this paper. Regarding the length of the paper, we avoid listing these consequences explicitly. We underline also that we give a simple form of Ulam stable results. It is easily improved in some other directions.

Author Contributions: All authors contributed equally and significantly to writing this article. All authors read and approved the final manuscript.

Acknowledgments: The authors thank the anonymous referees for their remarkable comments, suggestion and ideas that helped to improve this paper. The first and third authors would like to extend their sincere appreciation to the Deanship of Scientific Research at King Saud University for funding this group No. RG-1437-017.

Conflicts of Interest: The authors declare no conflict of interest. 


\section{References}

1. Doitchinov, D. On completeness in quasi-metric spaces. Topol. Appl. 1988, 30, 127-148. [CrossRef]

2. Romaguera, S.; Sanchis, M. Applications of utility functions defined on quasi-metric spaces. J. Math. Anal. Appl. 2003, 283, 219-235. [CrossRef]

3. Reilly, I.L. A Note on Quasi Metric Spaces. Proc. Jpn. Acad. 1976, 52, 428-430. [CrossRef]

4. Triebel, H. A new approach to function spaces on quasi-metric spaces. Rev. Mat. Complut. 2005, 18, 7-48. [CrossRef]

5. Sapto, I.; Diego, M.; Sharad, S. On the axiomatic approach to Harnack's inequality in doubling quasi-metric spaces. J. Differ. Equ. 2013, 254, 3369-3394.

6. Alsulami, H.; Karapinar, E.; Khojasteh, F.; Roldan, A. A proposal to the study of contractions in quasi metric spaces. Discrete Dyn. Nat. Soc. 2014, 269286. [CrossRef]

7. Aydi, H.; Felhi, A.; Karapinar, E.; Alojail, F.A. Fixed points on quasi-metric spaces via simulation functions and consequences. J. Math. Anal. 2018, 9, 10-24.

8. Sehgal, V.M. On fixed and periodic points for a class of mappings. J. Lond. Math. Soc. 1972, 5, 571-576. [CrossRef]

9. Guseman, L.F., Jr. Fixed point theorems for mappings with a contractive iterate at a point. Proc. Am. Math. Soc. 1970, 26, 615-618. [CrossRef]

10. Iseki, K. A generalization of Sehgal-Khazanchi's fixed point theorems. Math. Semin. Notes Kobe Univ. 1974, 2, 1-9.

11. Matkowski, J. Fixed point theorems for mappings with a contractive iterate at a point. Proc. Am. Math. Soc. 1977, 62, 344-348. [CrossRef]

12. Singh, K.L. Fixed-Point Theorems for Contractive-Type Mappings. J. Math. Anal. Appl. 1979, 72, $283-290$. [CrossRef]

13. Ray, B.K.; Rhoades, B.E. Fixed point theorems for mappings with a contractive iterate. Pac. J. Math. 1977, 71, 517-520. [CrossRef]

14. Kincses, J.; Totik, V. Theorems an counterexamples on contractive type mappings. Math. Balk. 1990, 4, 69-90.

15. Karapinar, E.; Roldan-Lopez-de-Hierro, A.-F.; Samet, B. Matkowski theorems in the context of quasi-metric spaces and consequences on G-metric spaces. Anal. Stiint. Ale Univ. Ovidius Constanta-Ser. Mat. 2016, 24, 309-333. [CrossRef]

16. Rus, I.A. Generalized Contractions and Applications; Cluj University Press: Cluj, Napoca, 2001.

17. Rus, I.A. The theory of a metrical fixed point theorem: Theoretical and applicative relevances. Fixed Point Theory 2008, 9, 541-559.

18. Khojasteh, F.; Shukla, S.; Radenović, S. A new approach to the study of fixed point theorems via simulation functions. Filomat 2015, 29, 1189-1194. [CrossRef]

19. Karapinar, E. Fixed points results via simulation functions. Filomat 2016, 30, 2343-2350. [CrossRef]

20. Karapinar, E.; Khojasteh, F. An approach to best proximity points results via simulation functions. J. Fixed Point Theory Appl. 2017, 19, 1983-1995. [CrossRef]

21. AAlharbi, S.S.; Alsulami, H.H.; Karapinar, E. On the Power of Simulation and Admissible Functions in Metric Fixed Point Theory. J. Funct. Spaces 2017, 2017, 2068163. [CrossRef]

22. Roldan, A.; Martinez-Moreno, J.; Roldan, C.; Karapinar, E. Coincidence point theorems on metric spaces via simulation functions. J. Comput. Appl. Math. 2015, 275, 345-355. [CrossRef]

23. Samet, B.; Vetro, C.; Vetro, P. Fixed point theorems for $\alpha \psi$-contractive type mappings. Nonlinear Anal. 2012, 75, 2154-2165. [CrossRef]

24. Karapinar, E.; Kumam, P.; Salimi, P. On $\alpha-\psi$-Meir-Keeler contractive mappings. J. Fixed Point Theory Appl. 2013, 2013, 94. [CrossRef]

25. Popescu, O. Some new fixed point theorems for $\alpha$-Geraghty-contraction type maps in metric spaces. J. Fixed Point Theory Appl. 2014, 2014, 190. [CrossRef]

26. Chen, C.-M.; Abkar, A.; Ghods, S.; Karapinar, E. Fixed Point Theory for the $\alpha$-Admissible Meir-Keeler Type Set Contractions Having KKM* Property on Almost Convex Sets. Appl. Math. Inf. Sci. 2017, 11, 171-176. [CrossRef]

27. Hammache, K.; Karapinar, E.; Ould-Hammouda, A. On Admissible Weak Contractions in b-Metric-Like Space. J. Math. Anal. 2017, 8, 167-180. 
28. Karapinar, E.; Samet, B. Generalized (alpha-psi) contractive type mappings and related fixed point theorems with applications. Abstr. Appl. Anal. 2012, 2012, 793486. [CrossRef]

29. Ali, M.U.; Kamram, T.; Karapinar, E. An approach to existence of fixed points of generalized contractive multivalued mappings of integral type via admissible mapping. Abstr. Appl. Anal. 2014, 141489. [CrossRef]

30. Aksoy, U.; Karapinar, E.; Erhan, I.M. Fixed points of generalized alpha-admissible contractions on b-metric spaces with an application to boundary value problems. J. Nonlinear Convex Anal. 2016, 17, 1095-1108.

31. Aydi, H.; Karapinar, E.; Zhang, D. A note on generalized admissible-Meir-Keeler-contractions in the context of generalized metric spaces. Res. Math. 2017, 71, 73-92. [CrossRef]

32. Arshad, M.; Ameer, E.; Karapinar, E. Generalized contractions with triangular $\alpha$-orbital admissible mapping on Branciari metric spaces. J. Inequal. Appl. 2016, 2016, 63. [CrossRef]

33. Alsulami, H.; Gulyaz, S.; Karapinar, E.; Erhan, I.M. Fixed point theorems for a class of alpha-admissible contractions and applications to boundary value problem. Abstr. Appl. Anal. 2014, 187031. [CrossRef]

34. Aydi, H.; Karapinar, E.; Yazidi, H. Modified F-Contractions via $\alpha$-Admissible Mappings and Application to Integral Equations. Filomat 2017, 31, 1141-1148. [CrossRef]

35. Almezel, S.; Chen, C.-M.; Karapinar, E.; Rakocevic, V. Fixed point results for various $\alpha$-admissible contractive mappings on metric-like spaces. Abstr. Appl. Anal. 2014, 2014, 379358.

36. Petru, T.P.; Petruşel, A.; Yao, J.-C. Ulam-Hyers stability for operatorial equations and inclusions via nonself operators. Taiwan. J. Math. 2011, 15, 2195-2212. [CrossRef]

37. Brzdek, J.; Popa, D.; Rasa, I.; Xu, B. Ulam Stability of Operators; Elsevier: Amsterdam, The Netherlands, 2018.

38. Qarawani, M. Hyers-Ulam Stability for Mackey-Glass and Lasota Differential Equations. J. Math. Res. 2013, 5, 34-40. [CrossRef]

39. Rus, I.A. Remarks on Ulam stability of the operatorial equations. Fixed Point Theory 2009, 10, 305-320.

40. Brzdek, J.; Karapinar, E.; Petrusel, A. A fixed point theorem and the Ulam stability in generalized dq-metric spaces. J. Math. Anal. Appl. 2018, 467, 501-520. [CrossRef]

41. Brzdek, J.; Popa, D.; Rasa, I. Hyers-Ulam stability with respect to gauges. J. Math. Anal. Appl. 2017, 453, 620-628. [CrossRef]

(C) 2018 by the authors. Licensee MDPI, Basel, Switzerland. This article is an open access article distributed under the terms and conditions of the Creative Commons Attribution (CC BY) license (http:/ / creativecommons.org/licenses/by/4.0/). 